\title{
Simultaneous red/green dual fluorescence detection of both the nuclear \& nucleolar compartments of live cells
}

\author{
J Coleman, Z Li, D Shen, D Gatica and W Patton
}

R\&D, Enzo Life Sciences, Farmingdale, NY 11735

The nucleolus represents a highly dynamic nuclear domain arising from an equilibrium between the level of ribosomal RNA synthesis and the efficiency of ribosomal RNA processing [1, 2]. Although the nucleolus is primarily associated with ribosome biogenesis, several lines of evidence now demonstrate that it has additional functions, such as regulation of mitosis, cell-cycle progression and proliferation, many forms of stress response and biogenesis of multiple ribonucleoprotein particles. Ribosome biogenesis is regulated throughout interphase and ceases during mitosis. Thus, there is a direct relationship between cell growth and nucleolar activities. Nucleoli are well known to be dramatically modified in cancer cells. Additionally, a large number of key proteins from both DNAand RNA-containing viruses are localized in the nucleolus, including the human immunodeciency virus (HIV)-1 Rev and Tat proteins. Targeting of viral proteins to the nucleolus not only facilitates virus replication, but may also be required for pathogenic processes. The nucleolus is also a sensor of stress due to the redistribution of the ribosomal proteins in the nucleoplasm through its disruption.

Historically, nucleolar imaging approaches have required laborious and time-consuming methods, such as microinjection of fluorescently-labeled RNA, fluorescence in situ hybridization (FISH) or use of fluorescent protein-tagged RNA-binding proteins (GFP or YFP constructs). A novel approach for highlighting nucleoli is through use of fluorescent RNA-selective molecular probes. However, while previously available RNA probes have provided satisfactory results in fixed cells, they performed relatively poorly in live cell applications. Additionally, the principle commercially available dye for this application emits in both the green and red regions of the visible light spectrum, limiting application in multicolor fluorescence microscopy applications. Since it is difficult to study nucleolar dynamics using fixed and permeabilized cells, a fluorescent, nucleolusselective live cell imaging dye is useful for the examination of dynamic changes in this organelle in relation to the organization of the DNA within the cell nucleus. Using a combination of a newly developed red-emitting DNA-intercalating dye and a newly developed green-emitting RNA-binding dye, we demonstrate simple dichromatic live-cell staining of the nucleus and nucleoli. The proprietary dyes exhibit minimal spectral overlap with one another, high signal intensity and low photobleaching. The RNA-binding dye shows maximal fluorescence signal within the nucleoli, and faint fluorescence throughout the nucleus. Weak fluorescence is also observed throughout the cytoplasm, predominantly associated with mitochondria. The red DNA intercalating dye maximally stains the DNA in the cell nucleus. Both dyes display high cellular plasma membrane and nuclear membrane permeability, and are well tolerated by living cells.

Using the dichromatic live-cell staining method we observed that the number of nucleoli in different mammalian cell types varied and nucleoli were of different sizes as well. There appeared to be an inverse relationship between the size and number of nucleoli in mammalian cells. For example, HeLa human cervical carcinoma cells typically displayed two prominent nucleoli per cell, as 
visualized by conventional fluorescence microscopy, while U-2-OS human bone osteosarcoma epithelial cells were observed to contain a half a dozen smaller nucleoli. In order to further benchmark the dichromatic staining method, changes in nucleolar dynamics were monitored using the antibiotic actinomycin D, an agent that suppresses DNA-directed RNA synthesis in vivo [3]. Typically, at higher doses of the drug ( $4-10 \mu \mathrm{g} / \mathrm{ml}$ for 4 hours), the green-fluorescence staining of the nucleolus in mammalian cells dissipated, or where still present, was dramatically reduced in amount, while at lower concentrations (1-4 $\mathrm{g} / \mathrm{ml}$ for 4 hours), a less dramatic reduction in nucleolar staining was observed. Using quantitative imaging, the half maximal effective concentration $\left(\mathrm{EC}_{50}\right)$ of actinomycin D was then estimated.

The dichromatic staining combination was found to be compatible with a broad range of fluorescence detection platforms, including conventional and confocal fluorescence microscopy as well as flow cytometry and high-content screening (HCS) instruments. Using a conventional fluorescence microscope, a "structured illumination" method [4] was employed in order to create high resolution optical sections thru the nucleus, facilitating three-dimensional reconstruction of the spatial relationship between the nucleoli and the nucleus. This demonstrated that in HeLa cells nucleoli penetrate significantly within the body of the nucleus, rather than simply being confined to its surface.

Potential applications for the dual detection strategy described herein include monitoring impaired ribosome biogenesis, inhibition of transcription, cell cycle dynamics and cellular stress, as well as, the distribution, trafficking and dynamics of nucleolar proteins, the distribution of viral proteins, and potentially as an aid to identify cancer cells. Drug toxicity screening on HCS platforms should also be feasible using the dichromatic staining approach.

\section{References}

[1] D. Hernandez-Verdun and P. Roussel Prog Cell Cycle Res.5: (2003) 301.

[2] V. Sirri, et al. Histochem Cell Biol. 129 (2008) 13.

[3] K. Smetana et al. Acta Histochem. 103 (2001) 325.

[4] M. Gustafsson Journal of Microscopy 198 (2000) 82. 